Sophia E. Schiza ${ }^{1}$, Winfried Randerath ${ }^{2}$, Manuel Sánchez-de-la-Torre ${ }^{3,4}$, Andrea Aliverti ${ }^{5}$, Maria R. Bonsignore ${ }^{6,7}$, Anita K. Simonds ${ }^{8}$, Pierantonio Laveneziana ${ }^{9,10}$

\title{
Editorial
}

\section{Continuous professional development: elevating sleep and breathing disorder education in Europe}

Sleep and breathing disorders are highly prevalent, representing a growing subspecialty of respiratory medicine. The term sleep disordered breathing (SDB) encompasses a range of conditions characterised by abnormal breathing during sleep, from chronic or habitual snoring, to frank obstructive sleep apnoea (OSA) or, in some cases, central sleep apnoea (CSA) and hypoventilation syndromes. OSA is the commonest form of SDB, leading to many potential consequences and adverse clinical outcomes, including excessive daytime sleepiness, impaired daytime function, metabolic dysfunction, and an increased risk of cardiovascular disease and mortality [1]. The estimated reported prevalence of moderate-to-severe SDB $\left(\geq 15\right.$ events $\left.\cdot h^{-1}\right)$ was $23.4 \%$ in women and $49.7 \%$ in men, and the prevalence of symptomatic OSA was $9 \%$ and $13 \%$, respectively [2]. However, in some populations, the prevalence of OSA is substantially higher, such as in patients been evaluated for bariatric surgery (estimated range $70-80 \%$ ), in patients who have had a transient ischaemic attack or stroke (estimated range 60-70\%) and in patients with cardiometabolic disease [3-6]. Limited data have been reported on CSA and non-obstructive sleep-related hypoventilation, which have received considerable interest in the sleep field within the past 10 years. Even if their prevalence was noted to be quite low relative to the prevalence of OSA [7], they are quite common in specific subpopulations [8-10].
Unquestionably, considerable progress has been made in past decades regarding basic science, and technical, clinical and therapeutic aspects of SDB. Furthermore, as sleep is essential for general health, SDB is important for general health as well. The field is still evolving and additional research to improve the diagnostic accuracy of simplified screening tools is needed to ensure that patients with SDB are accurately identified. However, the task of routine screening, assessment and management of SDB is particularly challenging. Inconsistencies in the diagnosis and management of SDB emphasise the need for standards in practice with special knowledge and expertise for medical practitioners managing respiratory sleep patients [11]. Moreover, in an era of evolving precision medicine, personalising therapeutic applications should be established on patient individualisation, correctly identifying their clinical profile and needs.

\section{The respiratory sleep medicine project}

Assembly 4 of the European Respiratory Society (ERS) is in the unique position of designing and delivering educational activities, such as successful symposia at the annual International ERS Congress, including Meet the Expert and Postgraduate Courses, research and education projects, Task Forces,
Cite as: Schiza SE, Randerath W, Sánchez-de-la-Torre M, et al. Continuous professional development: elevating sleep and breathing disorder education in Europe. Breathe 2020; 16: 190336.

@ERSpublications

The @EuroRespSoc launches a new sleep and breathing disorders continuous professional development programme http://bit.ly/30PU01P

\section{(1) \\ CrossMark}


Statements, Guidelines, research seminars and awards, which raise the standards of training for our respiratory health practitioners. Group 4.02, the largest in Assembly 4, provides a forum where scientists and clinicians interested in the causes and consequences of SDB can meet and share their ideas. By organising a specific Task Force, the Group has focused its activities on raising the awareness of sleep and breathing problems at the level of the European Commission and promoted identification of sleep-related disorders as a major priority in European Union health policies. Specifically, the Task Force produced Guidelines on non-continuous positive airway pressure (CPAP) therapies for obstructive sleep apnoea and Statements on the definition, discrimination, diagnosis and treatment of central breathing disturbances during sleep [12, 13]. Furthermore, upcoming statements are on the effectiveness of CPAP treatment on OSA in commercial and non-commercial motor vehicle drivers and new diagnostic techniques. Once published, the documents will provide clarity and direction for a number of topics in respiratory medicine with the aim of improving clinical practice. Moreover, the continuing educational interest of the ERS is shown by the number of issues of the ERS Monograph published in the past 20 years. The ERS Monograph is a quarterly book series covering a specific area of respiratory medicine and providing up-to-date and in-depth reviews that give clinicians a concise, comprehensive guide to symptoms, diagnosis and treatment. Group 4.02 also carries out educational and scientific activities in collaboration with the European Sleep Research Society (ESRS). In this direction, a collaborative European network project, the European Sleep Apnoea Database cohort, was established in 2007 with the objective to recruit a large prospective international cohort of patients with OSA for crosssectional and outcome research and to serve as a bridge for education and knowledge transfer between the various participating centres [14].

The ERS, in collaboration with the ESRS, established the biannual Sleep and Breathing Conference. The ESRS initiated an examination in sleep medicine at the 2012 ESRS Congress in Paris, France, leading to "somnologist" certification, followed by a second examination held during the 2013 Sleep and Breathing Conference in Berlin, Germany. So far, over 600 physicians, psychologists and sleep scientists across Europe have obtained this certificate. The certification confirms successful education and training in sleep medicine, including clinical scientific knowledge and technical skills and, therefore, proof of competence in the field of sleep medicine. Although National Sleep Societies (NSSs) have not adopted this qualification, many provide a certificate in sleep medicine in accordance with local customs or regulations. However, the Assembly of NSS and the ESRS Sleep Medicine Committee are in the process of identifying strategies to endorse this qualification, in order to achieve a homogenous standard of practice across Europe. The ESRS has also produced a catalogue of knowledge and skills as a basis for sleep medicine education and a multi-author textbook on sleep medicine, based on this catalogue, which was finalised in 2014. The ESRS is in the progress of updating this edition. Together the ERS and ESRS could significantly enhance the quality of each project with the objective to improve the quality of care for patients throughout Europe who suffer from these disorders.

\section{Periodical revisiting of the ERS curriculum development: future directions}

As no harmonised standards of training exist across Europe for respiratory sleep medicine, the ERS supported an application for a Sleep Harmonised Education in Respiratory Medicine for European Specialists (HERMES) Task Force project [15]. The HERMES Sleep Task Force and project were officially launched during the 2009 ERS International Congress in Vienna (Austria). The European core syllabus in respiratory disorders during sleep was published in 2011, followed by the production of educational materials including the ERS Handbook of Respiratory Sleep Medicine [16] and the respiratory sleep medicine curriculum in 2015. The ERS curriculum (formerly known as HERMES) was developed to present a common curriculum for medical and allied-health professionals, as well as to support teaching and certification in respiratory sleep medicine. The first course of the respiratory sleep certified training programme, initiated by the ERS Sleep Training Committee, took place during the 2017 Sleep and Breathing Conference(Marseille, France), followed by a second course held during the 2019 Sleep and Breathing Conference (Marseille), which was well attended. The aim of this course was to train respiratory professionals at a post specialty or postgraduate level in respiratory sleep medicine. The programme includes two parts with five independent modules and, following successful completion, a respiratory sleep certified training programme certificate is obtained. The programme overview can be viewed on the ERS website (www. ersnet.org/professional-development/ respiratory-sleep-certified-training-programme).

Over the years, the process of curriculum design has been improved within the ERS and it will continue to evolve, including more e-learning videos and CME online modules. There is also an updated edition of the ERS Handbook of Respiratory Sleep Medicine in planning. The sleep and breathing continuous professional development catalogue will be used as the contextual basis of our educational programme. This recently developed catalogue covers the SDB trajectory in 23 modules, as shown in table 1 . Each module 
Table 1 Module-based continuous professional development training

\section{Module 1: Neuroanatomy and neurobiology of sleep}

Regulation of sleep-wake cycle

Recognise basic neural processes and transmitters controlling wakefulness, non-rapid eye movement and rapid eye movement sleep states

Describe sleep architecture in a normal adult

Understand how the ageing process from fetal life to old age affects the sleep cycle

Sleep as a biologic process essential for life and optimal health

\section{Module 2: Physiology of sleep and breathing}

Control of breathing: explain factors that control breathing during sleep and wakefulness

Understand ventilatory response to hypercapnia, and hypoxaemia (including loop gain), arousal and apnoea threshold

Describe the functional anatomy of the upper airway

Discuss the factors that cause upper airway collapse during sleep

Relate control of breathing and upper airway function to the pathophysiology of obstructive sleep apnoea and central sleep apnoea

State how respiratory mechanics and ventilation are influenced by pathology

Explain sleep-related changes in respiratory mechanics in the aetiology of nocturnal hypoventilation

\section{Module 3: Cardiovascular and homeostatic mechanisms and sleep}

Understand how cardiovascular function, e.g. blood pressure and heart rate is affected during sleep and arousal from sleep

Appreciate impact of sleep on autonomic regulation and the consequences of sleep pathology...$g$. in OSA and CSA

\section{Module 4: Physiology of arterial blood gas and acid-base status}

The utility of arterial blood gas, capillary blood gas and venous blood gas

Diagnosis of A-B disorders: Henderson-Hasselbach equation, the relationship of oxygen tension, carbon dioxide tension and $\mathrm{pH}$

A-B disorders: the importance $\mathrm{D}(\mathrm{A}-\mathrm{a})$ difference, inspiratory oxygen fraction, alveolar gas equation and measuring oxygen shunts

\section{Module 5: Define SDB}

OSAHS, CSA, periodic breathing, Cheyne-Stokes respiration, obesity hypoventilation syndrome, nocturnal hypoventilation, upper airways resistance syndrome

Describe the epidemiology of OSAHS and CSA

Describe the different clinical phenotypes of OSA and their relationship to underlying pathophysiology

\section{Module 6: Assessment of the patient}

Sleep history to include:

Sleepiness and fatigue

Insomnia

Poor sleep quality

Nocturnal choking and gasping

Snoring

Morning headaches

Impotence

Low mood or labile mood

Cognitive impairment 
Table 1 Continued Module-based continuous professional development training

Signs:

Clinical examination of upper airway, nasal obstruction, tonsils and adenoids

Craniofacial disorders

Hypothyroidism

Obesity

Assess for comorbidities, e.g. COPD, chest wall disease, neuromuscular or neurological disorder, cardiovascular disease, endocrine or metabolic disorder

\section{Module 7: Evaluate impact of symptoms and establish pre-test probability of SDB}

Use of questionnaires: Epworth Sleepiness Scale, STOP-BANG score, Berlin questionnaire, 36-Item Short Form Health Survey, insomnia evaluation

Identify high-risk patients, e.g. severe sleepiness, unstable cardiac disease, nocturnal arrhythmia, baseline hypoxaemia, driving or other occupational risk

\section{Module 8: Diagnosis of respiratory sleep disorder}

Recognise the methodology of the different sleep tests (oximetry, respiratory polygraphy, full polysomnography)

Understand limitations of overnight oximetry, respiratory polygraphy and polysomnography, and home versus hospital-based sleep studies

Appreciate which patients to refer for polysomnography, e.g. those in whom diagnosis unclear on respiratory polygraphy, or poor treatment response or if non-respiratory sleep disorder suspected, e.g. narcolepsy, restless leg syndrome

Identify cases where further specialised examinations are needed, e.g. ear, nose and throat review of upper airway

Review cardiovascular, respiratory and metabolic disorders often associated with OSA

Clearly explain the high cardiometabolic risks associated with untreated OSA

Discuss the value of making improvements to lifestyle including weight loss, adherence to drug treatment for hypertension or diabetes, smoking cessation, alcohol reduction, physical exercise

\section{Module 9: Nocturnal capnography}

Role of capnography in patients with nocturnal hypoventilation

Understand limitations of overnight transcutaneous total carbon dioxide and end-tidal carbon dioxide tension monitoring

Appreciate use of capnography to establish ventilator settings

\section{Module 10: OSAHS management}

Distinguish mild, moderate and severe cases of OSA from normal results and upper airway resistance syndrome

Explain lifestyle interventions including weight loss, physical activity, exercise training, smoking cessation, alcohol reduction, avoidance of night sedation, sensible sleep hygiene measures

Understand role of ear, nose and throat intervention

Understand role and types of mandibular advancement splint or other oral device

Understand definition of positional sleep apnoea and role of positional devices

Appreciate indications for CPAP therapy

Understand differences between fixed level CPAP, variable CPAP and bi-level positive pressure therapy Depending on local pathways appreciate which patients to refer for these interventions

Understand CPAP related side-effects such as interface problems, airway drying, sleep disturbance Appreciate issues leading to poor adherence and how these may be addressed

Understand different ways of monitoring positive pressure therapy: clinic visits, data downloads from devices, telemonitoring 
Table 1 Continued Module-based continuous professional development training

Appreciate follow-up involves assessment of efficacy of therapy in controlling OSA but also impact on comorbidities and health-related quality of life

Importance of explaining rationale of treatment and likely outcomes to patient and advise on medico legal aspects, such as driving

Pre-peri and post-operative assessment and management of patients with OSAHS

Be aware of and follow local guidelines on diagnosis and management of OSAHS

\section{Module 11: CSA}

Classify aetiology of CSA: idiopathic, due to heart failure, cerebrovascular cause, e.g. cerebrovascular accident, opioid or other drug use, altitude

Differential symptoms and signs of OSA and CSA

Understand the pathophysiology of different types of CSA

Recognise which patients to refer for sleep studies

\section{Module 12: Management of CSA}

Understand impact of CSA on underlying pathology

CSA in chronic heart failure:

Appreciate importance of optimising therapy for heart failure

Understand role of CPAP in some patients including those with mixed OSA and CSA

Understand that ASV should not be used in heart failure patients with left ventricular ejection fraction $<45 \%$ Other forms of CSA:

Understand that CPAP or ASV may be of value in opioid-induced SDB

Appreciate further trials in progress assessing oxygen therapy in CSA and further role of ASV

\section{Module 13: Obesity hypoventilation syndrome}

Recognise symptoms, clinical presentation, and pathophysiology of obesity hypoventilation

Explain consequences (vascular disease, polycythaemia, cor pulmonale)

Assess indications for CPAP, NIV, application or supervision of mask and interface

Explain condition and the risks of obesity, and offer advice on how to decrease body mass index through physical activity, training and diet

Understand role of bariatric surgery, pre-operative assessment surgical risk, and peri-/post-operative management in OHS patients

\section{Module 14: Neuromuscular disorders}

Understand neuromuscular diseases causing respiratory failure

Neuromuscular conditions associated with respiratory muscle weakness

Appreciate methods to assess respiratory muscles weakness: spirometry, mouth pressures, peak cough flow, sniff inspiratory pressure, diaphragm EMG studies

Symptoms and signs of nocturnal hypoventilation and the probability of respiratory failure

Role of NIV versus invasive ventilation

Cough augmentation (cough assist) techniques

Use of NIV

Peri-operative assessment and management of neuromuscular disease patients

\section{Module 15: Chest wall disorder}

Recognise chest wall deformities causing respiratory failure, e.g. scoliosis, thoracoplasty

Symptoms and signs of nocturnal hypoventilation and the probability of respiratory failure, indications for sleep study

Role of NIV versus invasive ventilation, and cough augmentation (cough assist) techniques

Use of NIV

(Continued) 
Table 1 Continued Module-based continuous professional development training

\section{Module 16: Chronic respiratory failure}

Causes of respiratory failure

Principles of interpretation of blood gas analysis

National and international guidelines for treatment of acute and chronic hypercapnic respiratory failure Indications of additional oxygen treatment

Indications for long-term (home) mechanical ventilation in COPD, neuromuscular disease, chest wall disorders

\section{Module 17: Assisted ventilation}

Treatment of ventilatory failure

Describe types of positive pressure ventilation and different modes, e.g. bi-level positive airway pressure, volume ventilation, assured volume ventilation (AVAPS, iVAPS) ventilation, and other NIV modes; appreciate types of interface

Understand principles of titration of therapy with sleep studies and arterial blood gas measurement Recognise the concepts of compliance and adherence

Assessment of compliance and reasons for poor and good compliance

Understand indications for tracheostomy ventilation and who to refer for this

Understand NIV may have a palliative role, and importance of palliative therapy

Use of advance directives in end-stage diseases

\section{Module 18: Asthma/COPD and sleep}

Prevalence of SDB in asthma and COPD

Appraise symptoms, clinical presentation, pathophysiology and treatment of asthma, COPD

Relate the influence of comorbid respiratory disorders on breathing during sleep

Appreciate impact of drug therapy on sleep quality

Understand role of CPAP therapy in overlap syndrome (COPD+OSAHS) and asthma

\section{Module 19: Endocrine and metabolic disorders and SDB}

Appreciate increased prevalence of OSAHS in endocrine disorders (e.g. hypothyroidism), acromegaly metabolic disorders (e.g. diabetes mellitus, metabolic syndrome and renal failure)

Understand impact of treatment of OSAHS on underlying endocrine/metabolic disorder

\section{Module 20: Non-respiratory sleep disorders}

Consider presence of non-respiratory sleep disorders and referral for sleep specialist assessment Insomnia:

Recognise primary and secondary insomnia, management techniques including role of cognitive behavioural therapy and medication

Recognise presence of insomnia in some OSAHS phenotypes and its impact on implementation and adherence with therapy, e.g. CPAP

Other conditions to be aware of include restless leg syndrome

Narcolepsy, benign idiopathic hypersomnolence, parasomnias and associated conditions

Recognise which patients to refer for further investigation

\section{Module 21: Assessment of excessive sleepiness}

Understand the principles of sleep questionnaires, sleep diary, Multiple Sleep Latency Test, Maintenance of Wakefulness Test, Osler wake test in assessing excessive daytime somnolence in respiratory and non-respiratory sleep conditions, their advantages, imitations, and who to refer for these

\section{Module 22: Circadian disorders}

Understand principles of circadian rhythm and impact on sleep wake cycle across age range Appreciate impact of circadian disruption such as shift work and jet lag 
Table 1 Continued Module-based continuous professional development training

Understand interaction between shift work and SDB

Be aware of other circadian disorders such as delayed and advance sleep phase disorder

Appreciate role of interventions such as melatonin and bright light therapy

Actigraphy

Understand who to refer for actigraphy (and pros and cons of this investigation)

\section{Module 23}

Appreciate the medico-legal, societal and economic impact of respiratory sleep disorders, including implications of sleep disorders on driving and occupation

OSAHS: obstructive sleep apnoea-hypopnoea syndrome; ASV: adaptive servo-ventilation; NIV: noninvasive ventilation; AVAPS: average volume-assured pressure support; iVAPS: intelligent volumeassured pressure support.

will be maintained and regularly updated according to future developments by a faculty composed not only of senior scientific specialists in SDB, but also of committed Early Career Members since they represent our future. Digital and social media might be used to deliver the message and the education itself, utilising the already available ERS communication channels.

\section{Affiliations}

Sophia E. Schiza ${ }^{1}$, Winfried Randerath ${ }^{2}$, Manuel Sánchez-de-la-Torre ${ }^{3,4}$, Andrea Aliverti ${ }^{5}$, Maria R. Bonsignore $^{6,7}$, Anita K. Simonds ${ }^{8}$, Pierantonio Laveneziana ${ }^{9,10}$

${ }^{1}$ Sleep Disorders Unit, Dept of Respiratory Medicine, University General Hospital, Medical School of the University of Crete, Heraklion, Crete, Greece. .'Institute of Pneumology at the University of Cologne, Bethanien Hospital, Clinic for Pneumology and Allergology, Centre of Sleep Medicine and Respiratory Care, Solingen, Germany. . ${ }^{3}$ Group of Precision Medicine in Chronic Diseases, Hospital Arnau de Vilanova-Santa Maria, IRBLleida, Lleida, Spain. . ${ }^{4}$ Centro de Investigación Biomédica en Red de Enfermedades Respiratorias (CIBERES), Madrid, Spain. . ${ }^{5}$ Dipartimento di Elettronica, Informazione e Bioingegneria, Politecnico di Milano, Milan, Italy. . 6 Sleep and Chronic Respiratory Failure Clinic, University Hospital Paolo Giaccone, PROMISE Dept, University of Palermo, Palermo, Italy. . ${ }^{7}$ RIB, National Research Council (CNR), Palermo, Italy. ${ }^{8}$ Sleep and Ventilation Unit, Royal Brompton and Harefield NHS Foundation Trust, London, UK. . ${ }^{9}$ Sorbonne Université, INSERM, UMRS1158 Neurophysiologie respiratoire Expérimentale et clinique, Paris, France..$^{10} \mathrm{AP}$-HP Sorbonne Université, Groupe Hospitalier Pitié-Salpêtrière Charles Foix, Service des Explorations Fonctionnelles de la Respiration, de l'Exercice et de la Dyspnée du Département Médico-Universitaire "APPROCHES", Paris, France.

\section{Conflict of interest:}

S.E. Schiza has nothing to disclose. W. Randerath reports travel grants and speaking fees from Philips Respironics, Inspire, Resmed, Night Balance, Vanda Pharma and Bioprojet, outside the submitted work. M. Sánchez-de-la-Torre has nothing to disclose. A. Aliverti has nothing to disclose. M.R. Bonsignore has nothing to disclose. A.K. Simonds has nothing to disclose. P. Laveneziana reports personal fees from Novartis France and Boehringer France, outside the submitted work.

\section{References}

1. Spicuzza L, Caruso D, Di Maria G. Obstructive sleep apnoea syndrome and its management. Ther Adv Chronic Dis 2015; 6: 273-285.

2. Heinzer R, Vat S, Marques-Vidal P, et al. Prevalence of sleepdisordered breathing in the general population: the HypnoLaus study. Lancet Respir Med 2015; 3: 310-318.

3. Ravesloot MJ, van Maanen JP, Hilgevoord AA, et al. Obstructive sleep apnea is underrecognized and underdiagnosed in patients undergoing bariatric surgery. Eur Arch Otorhinolaryngol 2012; 269: 1865-1871.

4. Johnson KG, Johnson DC. Frequency of sleep apnea in stroke and TIA patients: a meta-analysis. J Clin Sleep Med 2010; 6: 131-137.

5. Punjabi NM. The epidemiology of adult obstructive sleep apnea. Proc Am Thorac Soc 2008; 5: 136-143.
6. Franklin KA, Lindberg E. Obstructive sleep apnea is a common disorder in the population - a review on the epidemiology of sleep apnea. J Thorac Dis 2015; 7: 1311-1322.

7. Donovan LM, Kapur VK. Prevalence and characteristics of central compared to obstructive sleep apnea: analyses from the Sleep Heart Health Study Cohort. Sleep 2016; 39: 1353-1359.

8. Ratz D, Wiitala W, Badr MS, et al. Correlates and consequences of central sleep apnea in a national sample of US veterans. Sleep 2018; 41: https://doi.org/10.1093/sleep/zsy058.

9. Baillieul S, Revol B, Jullian-Desayes I, et al. Diagnosis and management of central sleep apnea syndrome. Expert Rev Respir Med 2019; 13: 545-557.

10. Sateia MJ. International classification of sleep disordersthird edition: highlights and modifications. Chest 2014; 146 : 1387-1394. 
11. Mitchell S, Simonds A, Andreas S, et al. Introducing a core curriculum for respiratory sleep practitioners. Breathe 2015; 11: 50-56.

12. Randerath W, Verbraecken J, Andreas S, et al Definition, discrimination, diagnosis and treatment of central breathing disturbances during sleep. Eur Respir J 2017; 49 1600959

13. Randerath WJ, Verbraecken J, Andreas S, et al. NonCPAP therapies in obstructive sleep apnoea. Eur Respir J 2011; 37: 1000-1028.
14. Hedner J, Grote L, Bonsignore $M$, et al. The European Sleep Apnoea Database (ESADA): report from 22 European sleep laboratories. Eur Respir J 2011; 38 : 635-642.

15. De Backer W, Simonds AK, Horn V, et al. Sleep HERMES: a European training project for respiratory sleep medicine. Eur RespirJ 2011; 38: 496-497.

16. Simonds AK, de Backer W, eds. ERS Handbook of Respiratory Sleep Medicine. Sheffield, European Respiratory Society, 2012 